J. Austral. Math. Soc. (Series A) 42 (1987), 117-128

\title{
LIMIT THEOREMS FOR TRIANGULAR ARRAYS UNDER A RELAXED ASYMPTOTIC NEGLIGIBILITY CONDITION
}

\author{
MILOSLAV JIRINA
}

(Received 6 July 1984)

Communicated by T. C. Brown

\begin{abstract}
Let $\left\{X_{n k}\right\}$ be a triangular array of independent random variables satisfying the so-called tail-negligibility condition, i.e. such that $\operatorname{Prob}\left\{\left|X_{n k}\right|>a\right\} \rightarrow 0$ as both $k, n \rightarrow \infty$. It is also assumed that for each fixed $k, X_{n k}$ converges in distribution as $n \rightarrow \infty$. Theorems on the asymptotic behavior of the row sums of the array, analogous to those of the classical theory under the uniform negligibility condition, are presented.
\end{abstract}

1980 Mathematics subject classification (Amer. Math. Soc.): 60 F 05.

Keywords and phrases: triangular array of independent random variables, limit theorems for sums, tail negligibility.

\section{Direct theorems}

Let $\left\{X_{n k}\right\}, n=1,2, \ldots, k=1,2, \ldots, r_{n}, r_{n} \rightarrow \infty$, be a triangular array of random variables such that in each row the random variables are independent. The theory of limit distributions for $\sum_{k=1}^{r_{n}} X_{n j}$ is well established under the uniform negligibility (u.n.) condition, i.e. under the assumption that, for each $\alpha>0, \lim _{n} \max _{1 \leqslant k \leqslant r_{n}} \mathscr{P}\left(\left\{\left|X_{n k}\right|>a\right\}\right)=0$. In this paper we replace the u.n. condition by a milder condition called the tail-negligibility (t.n.) condition, i.e., by the assumption that, for each $a>0, \lim _{(k, n)} \mathscr{P}\left(\left\{\left|X_{n k}\right|>a\right\}\right)=0$, or, equivalently, that $\lim _{k} \sup _{n} \mathscr{P}\left(\left\{\left|X_{n k}\right|>a\right\}\right)=0$. (In this and similar relations, $\sup _{n}$ extends over all $n$ for which $X_{n k}$ is defined for a given $k$.) Under the u.n. condition, $X_{n k} \rightarrow 0$ in distribution for each $k$. We shall replace this by the assumption that, for each $k, X_{n k}$ converges in distribution as $n \rightarrow \infty$.

으 1987 Australian Mathematical Society $0263-6115 / 87 \$ A 2.00+0.00$ 
In this section we shall present two theorems on limit distributions for $\sum_{k=1}^{r_{n}} X_{n k}$ under our relaxed conditions, together with some examples. In Section 2, a converse theorem is presented. Some proofs may be found in Section 3.

Let $M_{n}, M$ be measures on $R=(-\infty, \infty)$. Consider the following two statements concerning an interval $I \subset R$ :

$$
\begin{gathered}
M_{n}(I)<\infty \text { for all } n, M(I)<\infty . \\
M_{n}(I) \rightarrow M(I) \text { if } I \text { is a continuity interval of } M .
\end{gathered}
$$

We shall say that $M_{n} \rightarrow M$ weakly, if (1) and (2) hold for all intervals, bounded or unbounded. We shall say that $M_{n} \rightarrow M$ weakly on bounded intervals, or b-weakly, if (1) and (2) hold for all bounded intervals. We shall say that $M_{n} \rightarrow M$ weakly on tail-intervals, or $t$-weakly, if (1) and (2) hold for all intervals $I$ separated from 0 , bounded or unbounded.

We shall use the following notation: For any set $A \subset R, \bar{A}$ will denote the complementary set $R-A$. The probability distribution (measure) of $X_{n k}$ will be denoted by $P_{n k}$. The measure $D_{n k}$ will be defined by $d D_{n k}(x)=x^{2} d P_{n k}(x)$. Our exposition will follow closely [1], XVII.7, and the same centring constants will be used, namely

$$
\nu_{n k}=\int_{-\infty}^{\infty} h(x) d P_{n k}(x)
$$

where $h$ is the function defined by $h(x)=x$ if $|x| \leqslant 1, h(x)=\operatorname{sgn} x$ if $|x| \geqslant 1$. As in the classical theory under the u.n. condition, any continuous bounded function behaving like $x$ near 0 may be used instead of $h$. Summing with respect to the second index $k$ will be reflected in the notation by an upper bar. So we shall write $\bar{X}_{n}=\sum_{k-1}^{r_{n}} X_{n k}, \bar{P}_{n}=\sum_{k=1}^{r_{n}} P_{n k}, \bar{D}_{n}=\sum_{k=1}^{r_{n}} D_{n k}, \bar{v}_{n}=\sum_{k=1}^{r_{n}} \nu_{n k}$.

Using our notation, we can now rewrite the two conditions mentioned above in the following way

(C1) (The t.n.-condition) For any $a>0$

$$
\lim _{k} \sup _{n} P_{n k}(\overline{[-a, a]})=0 .
$$

(C2) For each $k, P_{n k}$ converges weakly as $n \rightarrow \infty$ to a probability measure $P_{k}$.

It follows from (C2) that, for each $k, D_{n k}$ converges $b$-weakly as $n \rightarrow \infty$ to a measure $D_{k}$ defined by $d D_{k}(x)=x^{2} d P_{k}(x)$ and that, for each $k$,

$$
\nu_{n k} \underset{n}{\rightarrow} \nu_{k}=\int_{-\infty}^{\infty} h(x) d P_{k}(x)
$$


Also, if $\varphi_{n k}, \varphi_{k}$ are the characteristic functions of $P_{n k}, P_{k}$ respectively, then, for each $k, \varphi_{n k} \rightarrow \varphi_{k}$ uniformly on each $\left[-t_{0}, t_{0}\right]$.

The following is the main theorem of the paper.

THEOREM 1. Let (C1) and (C2) hold and let the following two assumptions be also satisfied:

(C3) $\bar{P}_{n}$ converges t-weakly to a measure $\bar{P}$.

(C4) $A$ finite limit

$$
\lim _{n}\left[\bar{D}_{n}([-a, a])-\sum_{k=1}^{r_{n}} \nu_{n k}^{2}\right]=\delta(a)
$$

exists for each $a>0$ such that both $\pm a$ are continuity points of $\vec{P}$.

Then $\bar{X}_{n}-\bar{\nu}_{n}$ converges in distribution, and the characteristic function of the limit distribution has the form $\Phi(t)=\Phi_{1}(t) \Phi_{2}(t)$, where

$$
\begin{gathered}
\Phi_{1}(t)=\prod_{k=1}^{\infty} e^{-i t v_{k}} \varphi_{k}(t), \\
\Phi_{2}(t)=\exp \left\{-\frac{1}{2} t^{2} \delta_{0}+\int_{-\infty}^{\infty}\left(e^{i t x}-1-i t h(x)\right) d P(x)\right\}, \\
\delta_{0}=\lim _{a \searrow 0}\left\{\delta(a)-\sum_{k=1}^{\infty}\left(D_{k}([-a, a])-\nu_{k}^{2}\right)\right\} \text { and } P=\bar{P}-\sum_{k=1}^{\infty} P_{k} .
\end{gathered}
$$

REMARKS. (a) The infinite product in (3) is convergent uniformly with respect to $t$ in any bounded interval, so that $\Phi_{1}$ is a proper characteristic function.

(b) If (C3) holds, then it is sufficient to check the existence of the limit $\delta(a)$ for one single $a>0$ in order to show that (C4) is also satisfied.

(c) It is easy to see that $\sum_{k=1}^{\infty} P_{k} \leqslant \bar{P}$, so that $P$ is a measure well defined on $(-\infty 0) \cup(0 \infty)$. The value $P(\{0\})$ is irrelevant.

(d) $\Phi_{2}$ is the characteristic function of an infinitely divisible distribution.

As in the classical theory under the u.n. condition, Theorem 1 can be simplified if all $\bar{X}_{n}$ have finite variances and if these variances converge to the variance of the limit distribution. If this is so, then it is natural to use the expectations rather than the constants $\nu_{n k}$ as centring constants, or to assume that the centring has been already done, i.e., that all $X_{n k}$ have zero expectations.

THEOREM 2. Let $(\mathrm{C} 1)$ and $(\mathrm{C} 2)$ hold and let the following two assumptions be also satisfied

(C5) For each $n, k, D_{n k}(R)<\infty, E\left(X_{n k}\right)=0$.

(C6) $\bar{D}_{n}$ converges weakly to a measure $\bar{D}$. 
Then $\bar{X}_{n}$ converges in distribution, and the characteristic function of the limit distribution has the form $\Phi(t)=\Phi_{1}(t) \Phi_{2}(t)$, where

$$
\begin{gathered}
\Phi_{1}(t)=\prod_{k=1}^{\infty} \varphi_{k}(t), \\
\Phi_{2}(t)=\exp \left\{\int_{-\infty}^{\infty} \frac{e^{i t x}-1-i t x}{x^{2}} d D(x)\right\}
\end{gathered}
$$

and $D=\bar{D}=\sum_{k=1}^{\infty} D_{k}$.

REMARK. The infinitely divisible part $\Phi_{2}$ can be written also in the form (4), if we put $\delta_{0}=\bar{D}(\{0\})=D(\{0\})$, and if we define the measure $P$ on $(-\infty 0) \cup(0 \infty)$ by $d P(x)=1 / x^{2} d D(x)$; alternatively, as (C6) implies (C3), $P$ can be defined also as in Theorem 1 .

We shall conclude this section with three examples. Trivial examples could be constructed such that, in each row, several random variables would contribute solely to $\Phi_{1}$ and the remaining solely to $\Phi_{2}$. In the examples presented here each $X_{n k}$ contributes both to $\Phi_{1}$ and $\Phi_{2}$.

In all three examples, $r_{n}=n$ and $z_{k}, k=1,2, \ldots$, is a sequence of positive numbers such that $\sum_{k=1}^{\infty} z_{k}^{2}<\infty$.

EXAMPLE 1. Each $X_{n k}$ assumes two values $z_{k}$ and $1 / \sqrt{n}$ with probabilities $\frac{1}{2}$. Applying Theorem 2 to $X_{n k}-E\left(X_{n k}\right)$, we get easily that $\bar{X}_{n}-\frac{1}{2}\left(\sqrt{n}+\sum_{k=1}^{n} z_{k}\right)$ converges in distribution, and the limit characteristic function is $\left(\prod_{k=1}^{\infty} \cos \frac{1}{2} z_{k} t\right)$. $e^{-1 / 8 t^{2}}$. If, in particular, $z_{k}=2^{-k}$, then the limit distribution is a convolution of the uniform distribution with a normal distribution.

EXAMPLE 2. Each $X_{n k}$ assumes four values $\pm z_{k}, \pm 1 / \sqrt{n}$ with probabilities $\frac{1}{4}$. Applying Theorem 1 or Theorem 2, we get that $\bar{X}_{n}$ converges in distribution, and the limit characteristic function is $\left(\prod_{k=1}^{\infty} \cos \frac{1}{2} z_{k} t\right)^{2} e^{-1 / 4 t^{2}}$. If, again, $z_{k}=2^{-k}$, then the limit distribution is a convolution of a triangular distribution and a normal distribution.

EXAMPLE 3. Each $X_{n k}$ assumes the values of $\pm z_{k}$ with equal probabilities $\frac{1}{2}\left(1-\frac{1}{n}\right)$ and the value 1 with probability $\frac{1}{n}$. This time the limit characteristic function for $\bar{X}_{n}$ is $\left(\Pi_{k=1}^{\infty} \cos z_{k} t\right) \exp \left(e^{i t}-1\right)$, i.e. the limit distribution is a convolution of the probability distribution represented by the infinite product and the Poisson distribution. 


\section{A converse theorem}

An interesting aspect of the classical theory under the u.n. condition is that it describes all possible limit distributions-namely, the infinitely divisible distributions - and provides necessary and sufficient conditions for the convergence in the distribution of $\bar{X}_{n}$. Under our more general conditions (C1) and (C2), the first problem is trivial; any probability distribution is clearly possible as a limit distribution. We shall therefore discuss only the second problem, i.e., the necessity of (C3) and (C4) for the convergence in distribution of suitably centered $\bar{X}_{n}$. The situation is slightly more complicated, as demonstrated by the next example.

EXAMPLE 4. Define three functions $\varphi^{(1)}, \varphi^{(2)}, \varphi^{(3)}$ in the following way:

$$
\begin{aligned}
& \varphi^{(1)}(t)=1-|t| \quad \text { if }|t| \leqslant 1, \quad \varphi^{(1)}(t)=0 \quad \text { if }|t| \geqslant 1, \\
& \varphi^{(2)}(t)=e^{-|t|} \quad \text { for all } t, \\
& \varphi^{(3)}(t)=e^{-|t|} \quad \text { if }|t| \leqslant 1, \quad \varphi^{(3)}(t)=e^{-1 / 2(1+|t|)} \quad \text { if }|t| \geqslant 1 .
\end{aligned}
$$

Then $\varphi^{(1)}$ and $\varphi^{(2)}$ are the characteristic functions of well known probability distributions, and $\varphi^{(3)}$ is also a characteristic function by the Polya theorem on symmetric convex functions; moreover, $\left[\varphi^{(2)}(t)\right]^{1 / n}$ and $\left[\varphi^{(3)}(t)\right]^{1 / n}$ are also characteristic functions. The triangular array $\left\{X_{n k}\right\}$ has $r_{n}=n+1$, and the probability distributions are defined in the following way: $X_{n 1}$ has the characteristic function $\varphi^{(1)}$ for each $n$; if $n$ is odd, $k=2,3, \ldots, n+1$, then $X_{n k}$ has the characteristic function $\left[\varphi^{(2)}\right]^{1 / n}$; if $n$ is even, $k=2,3, \ldots, n+1$, then $X_{n k}$ has the characteristic function $\left[\varphi^{(3)}\right]^{1 / n}$.

It is easy to see that $(\mathrm{C} 1)$ and $(\mathrm{C} 2)$ are satisfied and that $\bar{X}_{n}$ converges in distribution with the limit characteristic function $\varphi^{(1)} \varphi^{(2)}=\varphi^{(1)} \varphi^{(3)}$. On the other hand, if $n \rightarrow \infty$ through odd numbers, then $\bar{P}_{n} \underset{n}{\rightarrow} P_{1}+P$ t-weakly, where $P$ is the spectral measure of the infinitely divisible characteristic function $\varphi^{(2)}$; if $n \rightarrow \infty$ through even numbers, then $\bar{P} \rightarrow P_{1}+Q t$-weakly, where $Q$ is the spectral measure of $\varphi^{(3)}$. The measures $P$ and $Q$ must be different, and, therefore, (C3) does not hold.

This example shows that we must reformulate our problem if we want to make the conditions (C3) and (C4) necessary. Put, for each $k, \bar{X}_{n k}=\sum_{j=k}^{r_{n}} X_{n j}$ (so that $\left.\bar{X}_{n}=\bar{X}_{n 1}\right)$. It is easy to see that if (C1)-(C4) hold, then not only the sequence $\bar{X}_{n 1}-\bar{\nu}_{n}$, but also each sequence $\bar{X}_{n k}-\bar{\nu}_{n}$ (with $k$ fixed) converges in distribution as $n \rightarrow \infty$, and it turns out that under (C1) and (C2), the conditions (C3) 
and (C4) are also necessary for this kind of convergence. More exactly, we have the following theorem:

THEOREM 3. Let us assume that (C1) and (C2) hold and that there exist constants $\alpha_{n}$ such that $\bar{X}_{n k}-\alpha_{n}$ converges in distribution for each fixed $k$ as $n \rightarrow \infty$. Then (C3) and (C4) hold, and $\bar{\nu}_{n}-\alpha_{n}$ is a convergent sequence (so that $\alpha_{n}$ can be always replaced by the $\bar{\nu}_{n}$ used in Theorem 1$)$.

\section{Proofs}

As many details would be similar to those appearing in the theory under the u.n. condition, particularly to those in [1], XVII.7, we shall present a number of relations without a complete proof. In addition to the symbols introduced in Section 1 we shall use the following convention: a bar above a symbol provided with two lower indices $n, k$ indicates summation with respect to the second index in the original symbol between $k$ and $r_{n}$; thus $\bar{X}_{n k}=\sum_{j=k}^{r_{n}} X_{n j}, \bar{P}_{n k}=\sum_{j=k}^{r_{n}} P_{n j}$, etc.

To demonstrate the main ideas of the proofs of Theorems 1 and 2, we shall start with Theorem 2 rather than with Theorem 1, the proof of which is complicated by the necessary centring.

Proof of Theorem 2. We shall assume that (C1), (C2), (C5) and (C6) hold, and we shall use the following relations:

$$
\left.D_{n k} \text { converges weakly (as } n \rightarrow \infty\right) \text { to } D_{k} \text {, }
$$$$
\bar{D}_{n k} \text { converges weakly }(\text { as } n \rightarrow \infty) \text { to } \bar{D}-\sum_{j=1}^{k-1} D_{j} \text {, }
$$$$
\left.\bar{D}-\sum_{j=1}^{k-1} D_{j} \text { converges weakly (as } k \rightarrow \infty\right) \text { to } \bar{D}-\sum_{j=1}^{\infty} D_{j} \text {, }
$$

$$
\lim _{k} \sup _{|t| \leqslant t_{0}} \sup _{n}\left|\varphi_{n k}(t)-1\right|=0 \quad \text { for any } t_{0}>0,
$$

$$
\sup _{|t| \leqslant t_{0}} \sup _{n} \sum_{j=1}^{r_{n}}\left|\varphi_{n j}(t)-1\right|<\infty
$$

and

$$
\sup _{|t| \leqslant t_{0}} \sum_{j=1}^{\infty}\left|\varphi_{j}(t)-1\right|<\infty
$$

for any $t_{0}>0$. 
The relations (7), (8), and (9) follow easily from (C2) and (C6), and (10) follows from $(\mathrm{C} 1)$. To prove (11), we may use the inequality

$$
\left|\varphi_{n k}(t)-1\right| \leqslant \frac{1}{2} t_{0}^{2} D_{n k}(R) \text { for all }|t| \leqslant t_{0},
$$

which follows from

$$
\left(\varphi_{n k}(t)-1\right)=\int_{-\infty}^{\infty}\left(e^{i t x}-1-i t x\right) d P_{n k}(x) .
$$

Finally, (12) follows from (11) by (C2).

In the rest of the proof, $\ln z$ will denote the main branch of the natural logarithm of a complex number $z$ and we shall use it only for $|z-1|<1$, so that the usual power series development holds. In particular, if $|z-1| \leqslant \frac{1}{2}$, then

$$
\ln z=(z-1)+\alpha, \quad \text { where }|\alpha| \leqslant|z-1|^{2} .
$$

Using this, and (10) and (11), we see that to any $\varepsilon>0$ there corresponds $k_{0}$ such that

$$
\sup _{|t| \leqslant t_{0}} \sup _{k \leqslant k_{0}} \sup _{n}\left|\sum_{j=1}^{r_{n}} \ln \varphi_{n j}(t)-\sum_{j=k}^{r_{n}}\left(\varphi_{n j}(t)-1\right)\right|<\varepsilon .
$$

The proof of Theorem 2 will be concluded in three steps.

(a) By (14),

$$
\sum_{j=k}^{r_{n}}\left(\varphi_{n j}(t)-1\right)=\int_{-\infty}^{\infty} \frac{e^{i t x}-1-i t x}{x^{2}} d \bar{D}_{n k}(x),
$$

and, by (8) and (9),

$$
\lim _{k} \lim _{n} \sum_{j=k}^{r_{n}}\left(\varphi_{n j}(t)-1\right)=J(t) \quad \text { uniformly in }|t| \leqslant t_{0},
$$

where

$$
J(t)=\int_{-\infty}^{\infty} \frac{e^{i t x}-1-i t x}{x^{2}} d D(x) .
$$

Notice that $\exp J(t)=\Phi_{2}(t)$ is the characteristic function defined in Theorem 2.

(b) Take an $\varepsilon>0$. By (17),

$$
\left|\lim _{n} \sum_{j=k}^{r_{n}}\left(\varphi_{n j}(t)-1\right)-J(t)\right|<\varepsilon
$$

for all $|t| \leqslant t_{0}$ and all sufficiently large $k$. By $(12), \prod_{k=1}^{\infty} \varphi_{k}(t)$ is convergent uniformly in $|t| \leqslant t_{0}$, so that

$$
\left|\prod_{j=1}^{k-1} \varphi_{j}(t)-\prod_{j=1}^{\infty} \varphi_{j}(t)\right|<\varepsilon
$$

for all $|t| \leqslant t_{0}$ and all sufficiently large $k$. 
(c) Take a fixed $k_{0}$ satisfying simultaneously (15), (18) and (19) for all $|t| \leqslant t_{0}$. Then there exists $n_{0}$ such that, for all $n \geqslant n_{0}$ and all $|t| \leqslant t_{0}$, we have

$$
\begin{gathered}
\left|\sum_{j=k_{0}}^{r_{n}} \ln \varphi_{n j}(t)-J(t)\right|<2 \varepsilon \\
\left|\prod_{j=1}^{k_{0}-1} \varphi_{n j}(t)-\prod_{j=1}^{k_{0}-1} \varphi_{j}(t)\right|<\varepsilon .
\end{gathered}
$$

By (18),

$$
\left|\prod_{j=k_{0}}^{r_{n}} \varphi_{n j}(t)-\Phi_{2}(t)\right|<2 \varepsilon e^{2 \varepsilon},
$$

and, by (19), (21) and (22),

$$
\left|\prod_{j=1}^{r_{n}} \varphi_{n j}(t)-\Phi_{1}(t) \Phi_{2}(t)\right|<2 \varepsilon\left(1+e^{2} \varepsilon\right) .
$$

for all $n \geqslant n_{0}$ and all $|t| \leqslant t_{0}$, which proves the assertion of Theorem 2 .

Proof of Theorem 1. We shall assume that the assumptions (C1)-(C4) hold. We shall indicate only the changes that have to be made in the proof of Theorem 2. An auxiliary triangular array $X_{n k}^{*}=X_{n k}-\nu_{n k}$ will be used, and the corresponding symbols associated with $X_{n k}^{*}$ will be provided with *. So $P_{n k}^{*}$ will denote the probability distribution of $X_{n k}^{*}$, etc. In particular, $\nu_{n k}^{*}=\int_{-\infty}^{\infty} h(x) d P_{n k}^{*}(x)$.

Under (C1)-(C4), the array $X_{n k}^{*}$ satisfies similar relations, namely: $\lim _{k} \sup _{n} P_{n k}^{*}([\overline{-a, a}])=0$ for each $a>0$,

where

and

$$
\text { for each } \left.k, P_{n k}^{*} \text { converges weakly (as } n \rightarrow \infty\right)
$$
to a probability measure $P_{k}^{*}$,

$$
\bar{P}_{n}^{*} \text { converges } t \text {-weakly to a measure } \bar{P}^{*} \text {, }
$$

$$
\bar{P}^{*}=\bar{P}-\sum_{j=1}^{\infty} P_{j}+\sum_{j=1}^{\infty} P_{j}^{*}
$$

which, for each $a>0$, satisfies

$$
\bar{D}^{*}([-a, a])=\delta(a)-\sum_{j=1}^{\infty}\left(D_{j}([-a, a])-\nu_{j}^{2}\right)+\sum_{j=1}^{\infty} D_{j}^{*}([-a, a]) .
$$


The proofs of $\left(\mathrm{C} 1^{*}\right)-\left(\mathrm{C} 3^{*}\right)$ start with the formulas:

$$
P_{n k}^{*}([a, b])=P_{n k}\left(\left[a+v_{n k}, b+v_{n k}\right]\right)
$$

and

$$
\lim _{k} \sup _{n}\left|v_{n k}\right|=0 \text {, }
$$

which follows easily from (23) and ( $\mathrm{Cl})$. A starting point for $\left(\mathrm{C} 4^{*}\right)$ is the formula

$$
\begin{aligned}
D_{n k}^{*}([-a, a])= & D_{n k}\left(\left[-a+\nu_{n k}, a+\nu_{n k}\right]\right) \\
& -\nu_{n k}^{2}+2 \nu_{n k} \int_{\left[-a+\nu_{n k}, a+\nu_{n k}\right]} h(x) d P_{n k}(x) \\
& -\nu_{n k}^{2} P_{n k}\left(\left[-a+\nu_{n k}, a+\nu_{n k}\right]\right) .
\end{aligned}
$$

This holds for any $0<a<1$, for all $n$, and for all sufficiently large $k$ (such that $-1 \leqslant-a+\nu_{n k} \leqslant 1$, which is possible because of (24)).

The relations (8) and (9) will be replaced by

$$
\begin{aligned}
& \left.\bar{P}_{n k}^{*} \text { converges } t \text {-weakly (as } n \rightarrow \infty\right) \text { to } \bar{P}^{*}-\sum_{j=1}^{k-1} P_{j}^{*}, \\
& \bar{P}^{*}-\sum_{j=1}^{k-1} P_{j}^{*} \text { converges } t \text {-weakly (as } k \rightarrow \infty \text { ) to } P, \\
& \text { where } P \text { is the measure from Theorem } 1, \\
& \bar{D}_{n k}^{*} \text { converges } b \text {-weakly }(\text { as } n \rightarrow \infty) \text { to } \bar{D}^{*}-\sum_{j=1}^{k-1} D_{j}^{*},
\end{aligned}
$$

and

$$
\begin{aligned}
& \left.\bar{D}^{*}-\sum_{j=1}^{k-1} D_{j}^{*} \text { converges } b \text {-weakly (as } k \rightarrow \infty\right) \text { to a } \\
& \text { measure } D^{*} \text { such that } D^{*}(\{0\})=\delta_{0} \text {, where }
\end{aligned}
$$

$\delta_{0}$ is the number defined in Theorem 1.

They follow trivially from $\left(\mathrm{C} 2^{*}\right)-\left(\mathrm{C} 4^{*}\right)$.

The relations (10), (11), (12) and (15) also hold if $\varphi_{n k}$ is replaced by $\varphi_{n k}^{*}$. We shall call these relations $\left(10^{*}\right),\left(11^{*}\right),\left(12^{*}\right)$, and $\left(15^{*}\right)$, respectively. To prove them, (14) must be replaced by

$$
\varphi_{n k}^{*}(t)-1=i \nu_{n k}^{*} t+\int_{-\infty}^{\infty}\left(e^{i t x}-1-i t h(x)\right) d P_{n j}^{*}(x)
$$

and (13) by the inequality

$$
\left|\varphi_{n k}^{*}(t)-1\right| \leqslant t_{0}\left|\nu_{n k}^{*}\right|+\frac{1}{2} t_{0}^{2} D_{n k}^{*}([-1,1])+\left(1+t_{0}\right) P_{n k}^{*}([-1,1]),
$$

which holds for all $|t| \leqslant t_{0}$. The relation

$$
\sup _{n} \sum_{j=1}^{r_{n}}\left|p_{n j}^{*}\right|<\infty
$$

must be also used. It follows from $\left(\mathrm{C}^{*}\right)$ and from

$$
\left|\nu_{n k}^{*}\right| \leqslant 2\left|\nu_{n k}\right| P_{n k}\left(\overline{\left[-\frac{1}{2}, \frac{1}{2}\right]}\right) \text {, }
$$


which holds for all $n$ and all sufficiently large $k$ (such that $\left|\nu_{n k}\right| \leqslant \frac{1}{2}$ ). Another consequence of (32) and (C3*) is

$$
\lim _{n} \bar{\nu}_{n}^{*}=\sum_{j=1}^{\infty} \nu_{j}^{*}
$$

The series in (33) is absolutely convergent.

The proof of Theorem 1 may be concluded in three steps (a), (b), (c) similar to those in the proof of Theorem 2.

(a) By (29), we have

$$
\sum_{j=k}^{r_{n}}\left(\varphi_{n j}^{*}(t)-1\right)=i \bar{\nu}_{n k}^{*} t+\int_{-\infty}^{\infty}\left(e^{i t x}-1-i t h(x)\right) d \bar{P}_{n k}^{*} .
$$

Applying Theorem 2 of [1], XVII.2 to (34) twice, once with $n \rightarrow \infty$ and then with respect to $k \rightarrow \infty$, using (25)-(28) and (33), we get

$$
\lim _{k} \lim _{n} \sum_{j=k}^{r_{n}}\left(\varphi_{n j}^{*}(t)-1\right)=J(t) \quad \text { uniformly in }|t| \leqslant t_{0},
$$

where $J(t)=-\frac{1}{2} \delta_{0} t^{2}+\int_{-\infty}^{\infty}\left(e^{i t x}-1-i t h(x)\right) d P(x)$. Hence, $\exp J(t)=\Phi_{2}(t)$ is the characteristic function defined in Theorem 1.

The steps (b) and (c) would be virtually the same as in the proof of Theorem 2, but with $\varphi_{n k}$ replaced by $\varphi_{n k}^{*}$ and (17) replaced by (35).

Proof of Theorem 3. We shall demonstrate only the essential part of the proof.

(a) We shall first assume that all assumptions of Theorem 3 are satisfied and that $\left(11^{*}\right)$ also holds (although, in the prevoius proof, $\left(11^{*}\right)$ was the consequence of $\left(\mathrm{C}^{*}\right)$, which we do not assume now). Under these assumptions, $\left(10^{*}\right)$ and $\left(15^{*}\right)$ also hold.

Let us denote the characteristic function of $\bar{X}_{n k}-\alpha_{n}$ by $\psi_{n k}(t)$, so that

$$
\psi_{n k}(t)=\exp \left(i t\left(\bar{\nu}_{n k}-\alpha_{n}\right)\right) \cdot \prod_{j=k}^{r_{n}} \varphi_{n j}^{*}(t) .
$$

Take a fixed $t_{0}>0$. Because of $\left(10^{*}\right)$, there exists $k_{0}$ such that $\ln \varphi_{n j}^{*}(t)$ is well defined for all $|t| \leqslant t_{0}$, all $n$ and all $k \geqslant k_{0}$. The function

$$
\lambda_{n k}(t)=i t\left(\bar{\nu}_{n k}-\alpha_{n}\right)+\sum_{j=k}^{r_{n}} \ln \varphi_{n j}^{*}(t)
$$

is then the continuous logarithm of $\psi_{n k}(t)$ (such that $\lambda_{n k}(0)=0$ ). It follows from the assumptions of Theorem 3 that, for each $k, \psi_{n k}$ is uniformly convergent on $\left[-t_{0}, t_{0}\right]$ as $n \rightarrow \infty$. Hence, for each $k \geqslant k_{0}, \lambda_{n k}$ converges uniformly on $\left[-t_{0}, t_{0}\right]$ 
as $n \rightarrow \infty$. We are going to show that the sequence of functions $J_{n}$ defined by

$$
J_{n}(t)=i t\left(\bar{\nu}_{n}-\alpha_{n}\right)+\sum_{k=1}^{r_{n}}\left(\varphi_{n k}^{*}(t)-1\right)
$$

is also convergent uniformly on $\left[-t_{0}, t_{0}\right]$. For a given $\varepsilon>0$, choose $k_{0}$ so that $\left(15^{*}\right)$ holds. Then

$$
J_{n}(t)=\rho_{n}(t)+\delta_{n}(t),
$$

where $\rho_{n}(t)=\lambda_{n k_{0}}(t)+\sum_{j=1}^{k_{0}-1}\left[i t \nu_{n j}+\varphi_{n j}^{*}(t)-1\right]$, and where $\left|\delta_{n}(t)\right|<\varepsilon$ for all $n$ and all $|t| \leqslant t_{0}$. Because $\lambda_{n k_{0}}(t)$ converges uniformly in $|t| \leqslant t_{0}$ as $n \rightarrow \infty$, and because (C2) and (C2*) hold, there exists $n_{0}$ such that $\left|\rho_{m}(t)-\rho_{n}(T)\right|<\varepsilon$ for all $m, n \geqslant n_{0}$ and all $|t| \leqslant t_{0}$. Then $\left|J_{m}(t)-J_{n}(t)\right|<3 \varepsilon$ for all $m, n \geqslant n_{0}$ and all $|t| \leqslant t_{0}$. Notice that it is in this part of the proof where the assumption that $\bar{X}_{n k}-\alpha_{n}$ converges in distribution as $n \rightarrow \infty$ for each $k$, rather than just for $k=1$, is used. The functions $J_{n}$ can be rewritten in the form

$$
J_{n}(t)=i t\left(\bar{\nu}_{n}-\alpha_{n}+\bar{\nu}_{n}^{*}\right)+\int_{-\infty}^{\infty}\left(e^{i t x}-1-i t h(x)\right) d \bar{P}_{n}^{*}(x) .
$$

Hence, by Theorem 2 of [1], XVII.2, the conditions $\left(C 3^{*}\right),\left(\mathrm{C} 4^{*}\right)$ are satisfied, and the sequence $\bar{\nu}_{n}-\alpha_{n}+\bar{\nu}_{n}^{*}$ is convergent. We can see from the discussion of the proofs of $\left(\mathrm{C}^{*}\right)$ and $\left(\mathrm{C}^{*}\right)$ that $\left(\mathrm{C} 3^{*}\right)$ implies $(\mathrm{C} 3)$ and that, under $\left(\mathrm{C} 3^{*}\right)$, (C4*) implies (C4). Further, (33) holds under (C3*), so that $\bar{\nu}_{n}-\alpha_{n}$ is convergent. This concludes the proof of Theorem 3 under the additional assumption that $\left(11^{*}\right)$ holds.

(b) To show that the assumption $\left(11^{*}\right)$ may be dropped, i.e. that the assumptions of Theorem 3 imply $\left(11^{*}\right)$, the symmetrization procedure used in [1], XVII.7 may be applied. We shall not elaborate on the details.

\section{Related papers}

In [3] necessary and sufficient conditions are given for $\bar{X}_{n}$ to be asymptotically normal without the assumption of asymptotic negligibility. In [2], the method of [3] is generated, admitting limit distributions belonging to Linnik's $I_{0}$-class of infinitely divisible distributions. Within this restriction on the limit distribution, the theorems of [2] are more general than those of the present paper, as a kind of relaxed asymptotic negligibility is a part of both the necessary and sufficient condition. On the other hand, as a sufficient condition, Theorems 1 and 2 of the present paper are more general because they do not impose any restriction on the 
limit distribution, and are constructive in the sense that, contrary to the theorem of [2], they provide an algorithm for finding the limit distribution in concrete cases.

\section{References}

[1] W. Feller, An Introduction to Probability Theory and Its Applications, Vol. II (2nd ed., Wiley, New York, 1971).

[2] Y. Y. Machis, 'Non-classical limit theorems', Theory Probab. Appl. 16 (1971), 175-182.

[3] V. M. Zolotarev, 'A generalization of the Lindeberg-Feller theorem,' Theory Probab. Appl. 12 (1967), 608-618.

School of Mathematical Sciences

The Flinders University of South Australia

Bedford Park, S. A. 5042

Australia 